\title{
Telarquia Prematura
}

\author{
Dra. Ximena Cuello A. ; Dra. Ximena Yivanco. W. ${ }^{2}$; \\ Dra. Nelly Abodovsky G. ${ }^{1-2}$
}

Premature thelarche

Fourty eight cases of premature thelarche are analyzed. Patient's ages ranged from 1 month to 8 years. A detailed history and phisical examination, anthropometric measurements, breast development evaluation (According to Tanner classification), $x$ - ray examination of the skull, radiological evaluations for bone age, electroencephalogram (EEG), funduscopic examination, urinary 17-ketosteroids, 17 hidroxycorticoids, gonadotropins and vaginal cytology were done. Follicle-stimulating hormone (FSH), Luteinising hormone (LH) and Prolactin (PRL) were also mesured by radioinmunoassay. All the girls were followed by periods of 3 months to 6.5 years (mean 2.5 years). Premature breast development was related with the presence of vaginal estrogenism in more than $50 \%$ of the cases. Aoceleration of bone age was infrecuently found. Plasma concentrations of FSH and LH were in normal ranges in most of, the cases. No change in growth velocity was observed. We found only slight and not significant displacements of the rapid growth period to earlier ages in girls of older ages at the beggining of thelarche. No progression of prema ture telarche to precocious puberty was observed among our patients.

(Key words: Thelarche, premature. Gonadotropines. FSH and LH production. Bone age. Vaginal cy tology).

Telarquia prematura es la aparición de desarrollo mamario antes de los 8 años de edad, sin otro signo de maduración sexual ${ }^{1}$. Su hallazgo es relativamente frecuente y plantea el diagnóstico diferencial con pubertad precoz verdadera, o pseudo pubertad precoz. Su patogenia no ha sido aún aclarada. Grümbach y Kaplan ${ }^{2-3}$ sitúan el problema en el órgano efector, que presentaría una mayor sensibilidad a las concentraciones "fisiológicas" de estrógenos circulantes; otros autores piensan que sería causada por sobreproducción autónoma de estrógenos a partir de folículos ováricos que habrian experimentado transformación quística $y$ luteinización ${ }^{3-4}$; un tercer grupo postula que existiria un aumento transitorio del factor liberador de hormona luteinizante (LHRH) con mayor respuesta de la hormona folículo estimulante (FSH) $^{5-6}$.

El objetivo de nuestro trabajo fue determinar si la telarquia prematura representa un fenómeno aislado a si constituye la primera manifestación de pubertad precoz, y establecer si su aparición se asocia con alguna variación del ritmo de cre. cimiento. Para ello se analizaron los casos de telarquia prematura que consultaron en el Hospital San Juan de Dios, entre los años 1975 a 1981.

1 Servicio de Pediatría, Departamento de Endocrinología Infantil, Hospital San Juan de Dios.

2 Departamento de Pediatría, División Occidente. Facultad de Medicina. Universidad de Chile.

\section{MATERIAL Y METODO}

Se estudiaron 48 nifias cuya edad fluctuaba entre 1 mes y 8 aftos y se distribuyeron en cuatro grupos etarios con intervalos de 2 años cada uno. En todos los casos se tomó historja clínica exhaustiva para descartar exposición a estrógenos.

Las pacientes fueron controladas por periodos que variaron entre 3 meses y 6 anos 6 meses, đesde el momento en que se hizo el diagnóstico: En 36 casos la observación fue mayor que 6 meses, y en 12 menor. El grado y la evolución del desarrollo mamarios fueron evaluados según Tanner? .

Para calificar la evolución de la telarquia, se usó el criterio de Kaplan y Cols. ${ }^{2}$, que la identifica como lndeterminada,cuando el período de observación es menor de 3 meses; Estacionaria, si no se observan cambios en 3 o más meses de observación; Progesiva, si continúa el desarrollo mamario y Regresiva, cuando disminuye o desaparece ta telarquia.

Se efectuaron mediciones radiológicas de edad ósea (EO), Radiografias de cráneo, Colpocitograma funcional-usando el índice de Kaufman y Leeds- ${ }^{9-10-11}$, mediciones plasmáticas de hormonas FSH (MRC 69/104), luteinizante (LH) (MRC 68/40) y prolactina (PRL) (MRC 75/504) por radioinmunoensayo (RIA). Se midieron, en orina de 24 hrs., Gonadotrofinas biológicas (G.T.), 17 hidroxicorticoides $(170 \mathrm{H})^{21-22}, 17$ ketoesteroides $(17 \mathrm{ks})^{23}$. En 10 niñas se efectuó electroencefalogramas (EEG) y en 12 fondo de ojos. 
La velocidad de crecimiento, es decir, el incremento de talla por año cronológico, se tabuló con la edad ósea, de acuerdo a los criterios enunciados por Bossi y cols. ${ }^{13}$. El cuociente de desarrollo (C.D.), que es la relación entre la variación de la edad estatural (EE) y la variación de la edad ósea (EO) en un mismo período, fué calculado mediante la fórmula $C D=\triangle \mathrm{EE}$. El való nórmal $\triangle \overrightarrow{\mathrm{EO}}$

es igual a 1 ; un valor superior indica un buen pronóstico de talla final; un valor inferior a 1 implica deterioro de crecimiento ${ }^{\mathbf{1 3}}$.

Para el análisis del conjunto de antecedentes relativos a E.O. versus Edad cronológica y Gonadotrofinas biológivas versus FSH y LH, se aplicó el método de correlación lineal ${ }^{19}$.

\section{RESULTADOS}

Las 48 niñas con telarquia prematura se dis. tribuyeron acumulándose de preferencia entre las edades inferiores a 2 años $(n=10)$, y 6 a 8 años $(n=22)$. Sólo 7 pacientes tenían 2 a $<4$ años y 3 entre 4 y $<6$ años.

El extendido vaginal practicado en 34 casos, mostró en 20 casos $(58,8 \%)$ presencia de estrogenismo en grado variable: 11 leve $(+), 5$ moderado $(++), 2$ franco $(+++)$ y 2 marcados $(++++)$ y ausencia de efecto estrogénico en $14(41,2 \%)$. (Fig. 1).

En los 20 casos con estrogenismo yaginal, 11 tenían edad ósea normal ( $65 \%$ ) y 7 edad ósea adelantada ( $35 \%$ ). En 13 de 14 casos sin estrogenismo vaginal se midió la edad ósęa que en 12 resultó normal $(92 \%)$ y en otro adelantada.

En todas las pacientes se relacionó la edad ósea con la edad cronológica; en la mayoría ambos parámetros fueron concordantes con un range de variación normal de más o menos 6 meses (coeficientes correlación lineal $(\mathrm{EO} / \mathrm{E} \mathrm{Cr}$ ), $r=0,9655$ (Fig. 2). En 7 casos existía un adelanto de la edad ósea: Para establecer si en ellos había deterioro de la talla, se calculó el cuociente de desarrollo (CD), que en 3 aiñas fue inferior a 1 pero tendió a corregirse durante el período de observación: los otros 4 tenian CD inicialmente bueno que se deterioró con el transcurso del tiempo en 2 y no presentó variación en los restantes (Fig. 3).

La evolución de la telarquia se observó por separado en los 4 grupos etarios (Fig. 4). En los 3 primeros, con un total de 26 ninas menores de 6 afros, la evolución de la telarquia fue estacionaria en 12, regresiva en 9, progresiva en 2 e indeterminada en 3 . En el grupo de 6 a 8 antos (22 niñas) la evolución fue estacionaria en 4 , regresiva en 9, progresiva en $7, e$ indeterminada en 2 .

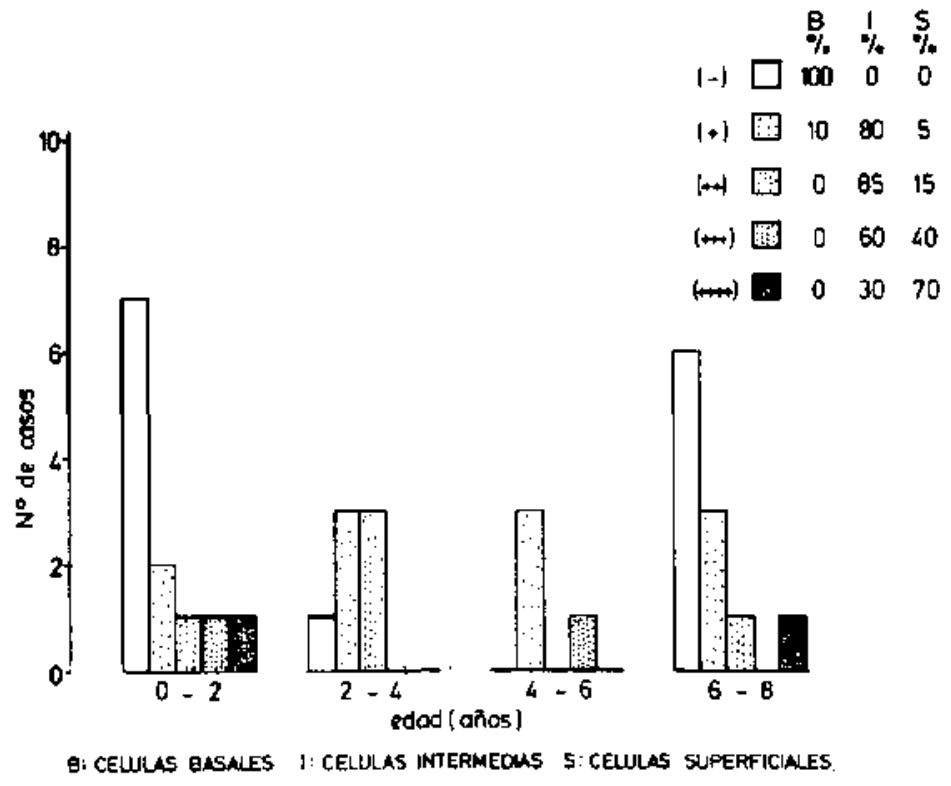

Figura 1.

Magnitud del Estrogenismo en 34 casos de Telarquja Prematura, según edad. En el extremo superior izquierdo se expresan los criterios de calificación según la distribución porcentual de los distintos tipos de células. 


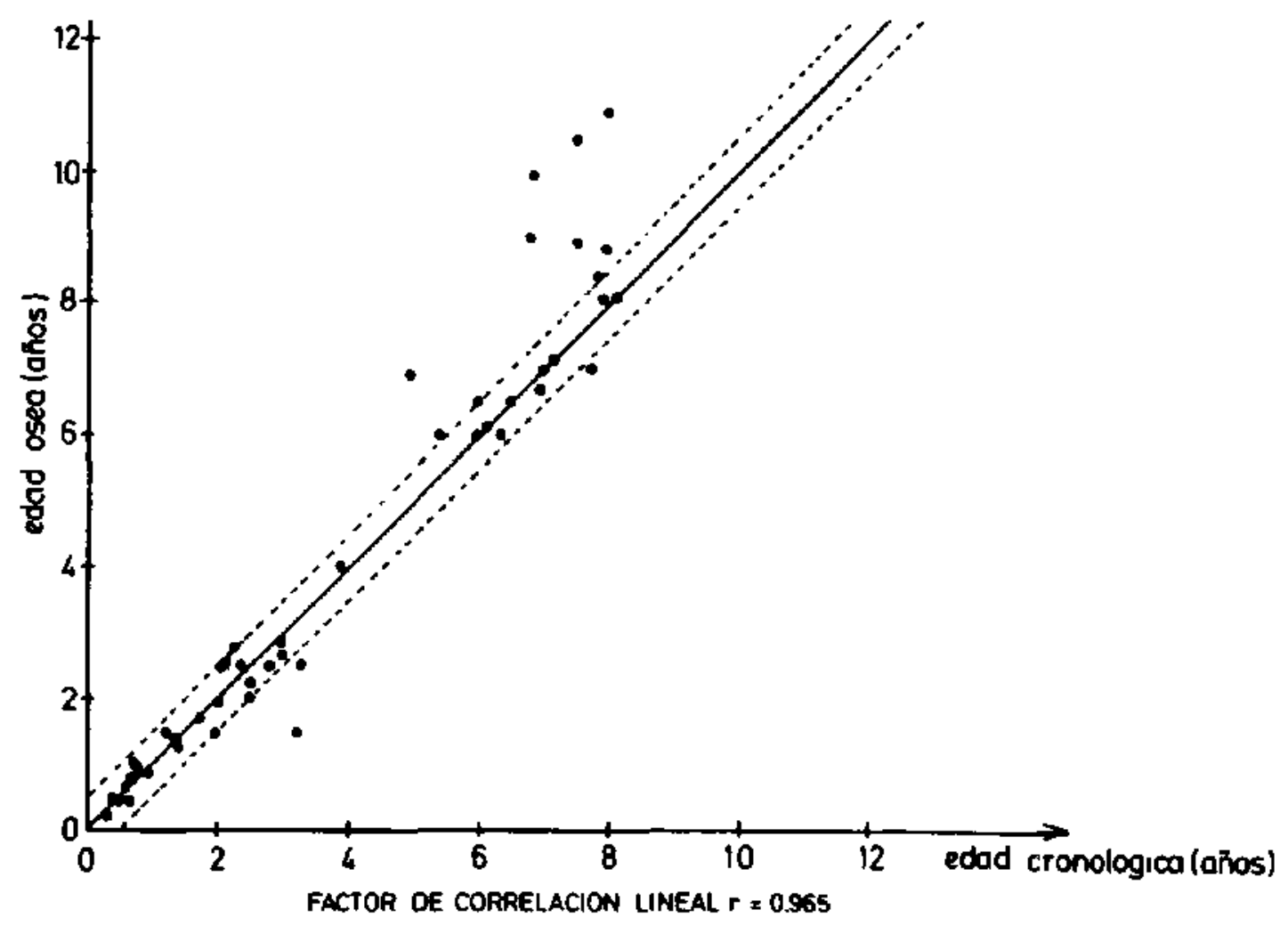

Figura 2.

Relación edad ósea, edad cronológica en 48 casos de Tejarquia Prematura $(\mathrm{r}=0,965)$.
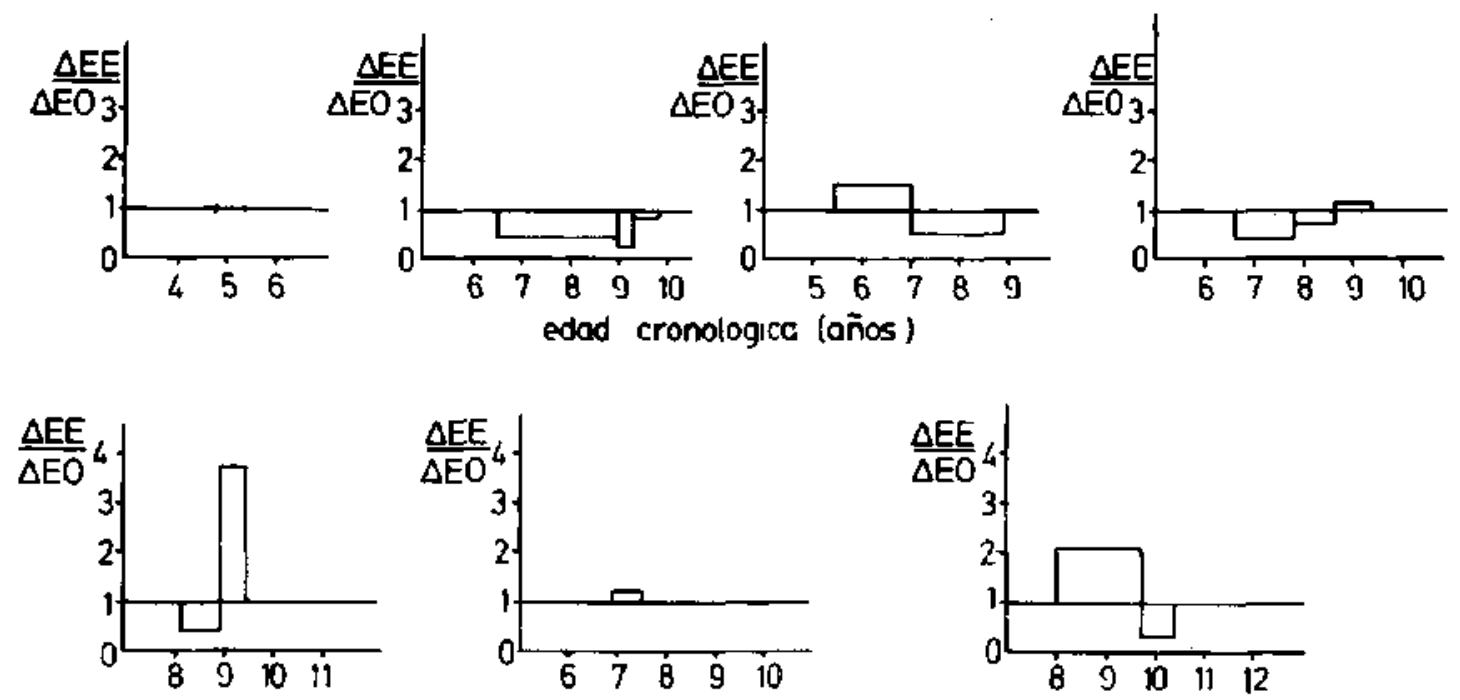

edad cronologıca (años)

Figura 3.

Evolución del cuociente de desariollo en los ? pacientes con Telarquia Prematura y edad ósea adelantada. $\triangle E F / \triangle E O=$ Cuociente de desarroilo (Normal $=1$; ver texto). in tres casos el cuociente de desarrollo tiende a mejorar. in dos es inicialmente bueno $y$ se deteriora y en utros dos no varía. 


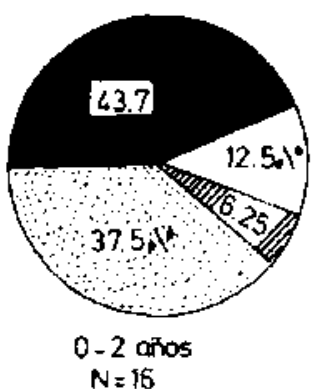

$N=16$

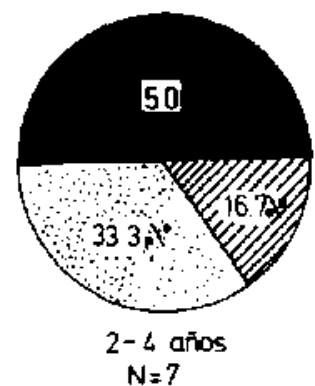

$N=7$
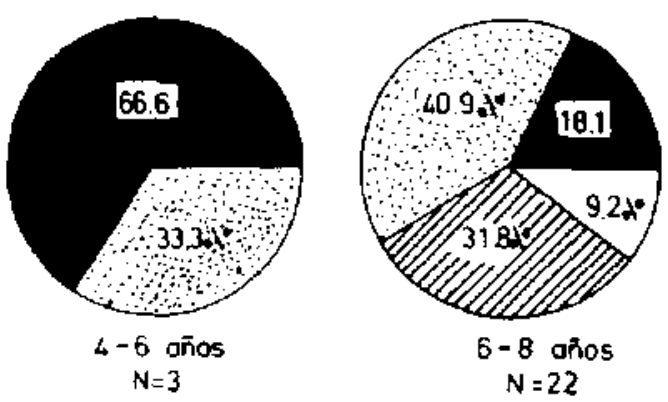

Figura 4.

Tipos de evolución del desarrollo mamario en 48 casos de Telarquia Prentatura según edad, observados durante un período promedio de 2.5 años.

El comportamiento de los casos con evolución progresiva, en las dos pacientes menores de 6 años se caracterizó por un cambio de desarrollo mamario de $\mathrm{B}_{2}$ a $\mathrm{B}_{3}$. De las 7 mayores de 6 años, 2 sólo mostraron progresión del desarrollo mamario $\mathrm{deB}_{2}$ a $\mathrm{B}_{3}$ sin otras manifestaciones: las otras 5 , cuya evolución se observó por periodos de 2,5 a 5 anos, presentaron iniciación del vello pubiano entre los 9 años 7 meses y los 10 años 6 meses; en dos de ellas ocurrió la menarquia a $\operatorname{los} 11$ años y 11 años 4 meses de edad respectivamente.

El estudio comparativo de Ja telarquia con el estrogenismo vaginal y la maduración ósea mostró que en 6 casos de telarquia tegresiva, 5 te. nian estrogenismo leve $(+)$ y uno moderado $(++)$, con edad osea normal en 5 y adelantada en uno. En 9 casos con telarquia estacionaria, había 4 con estrogenismo leve $(t), 2$ moderado $(++), 2$ marcado (+++) y uno franco (++++), mientras la edad ósea era normal en 7 , adelantada en uno y atrasada en otro. En 4 niñas con telarquia de evolución progresiva, 2 tenian estrogenismo leve (+) y 2 moderado. La edad ósea era normal en 2 y adelantada en 2 . (Fig. 5).

En 24 pacientes se hicieron mediciones plasmáticas de FSH y LH: En 16 ambos valores es. taban dentro del rango normal, 9 niñas tenían el FSH $(n=5)$ o el LH $(n=4)$ por encima del valor máximo normal. En un caso ambas hormonas estaban concomitantemente elevadas $y$ en otro habia aumento de LH con FSH normal (Fig. 6).

En los 9 casos con aumento de FSH, LH o ambos en el plasma, se analizó el comportamien- to del estrogenismo vaginal y la maduración ósea. En 5 con edad ósea normal, habian 2 con estrogenismo leve a moderado y $3 \sin$ estrogenismo. En 2 casos con edad ósea adelantada, uno tenía colpocitograma con estrogenismo leve y en el otro no se determinó. En 2 casos no se evaluó da edad ósea.

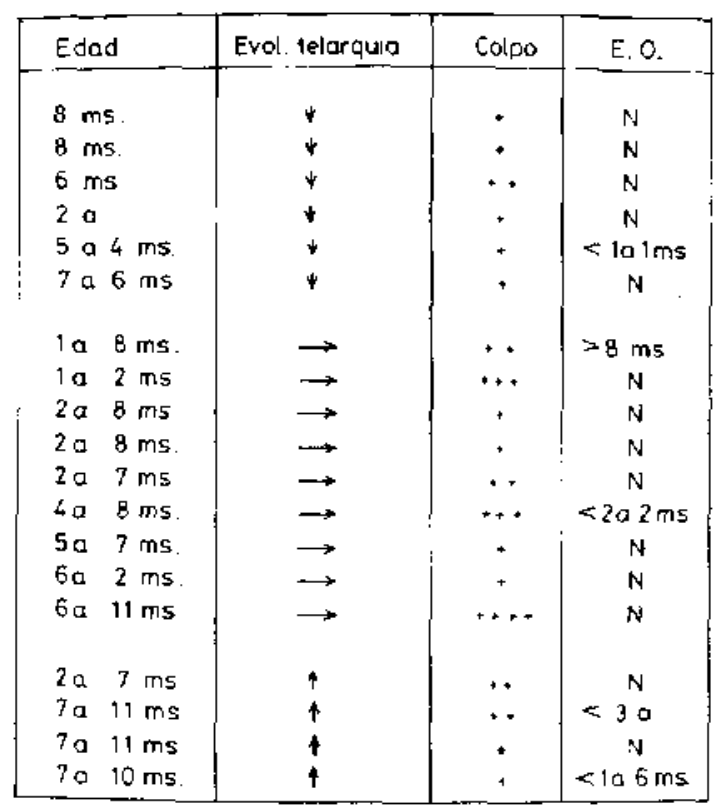

Figura 5.

Rclación entre la evolución de la Telarquia, el grado de estrogenismo vaginal (colpocitograma) y la cdad ósea ( $E O)$ en 48 casos de Telarquia Pientatura.

Lvolución regresiva $(\downarrow)$; evolución cstacionaria $\rightarrow$; evolución progrestiva $(\uparrow)$. 

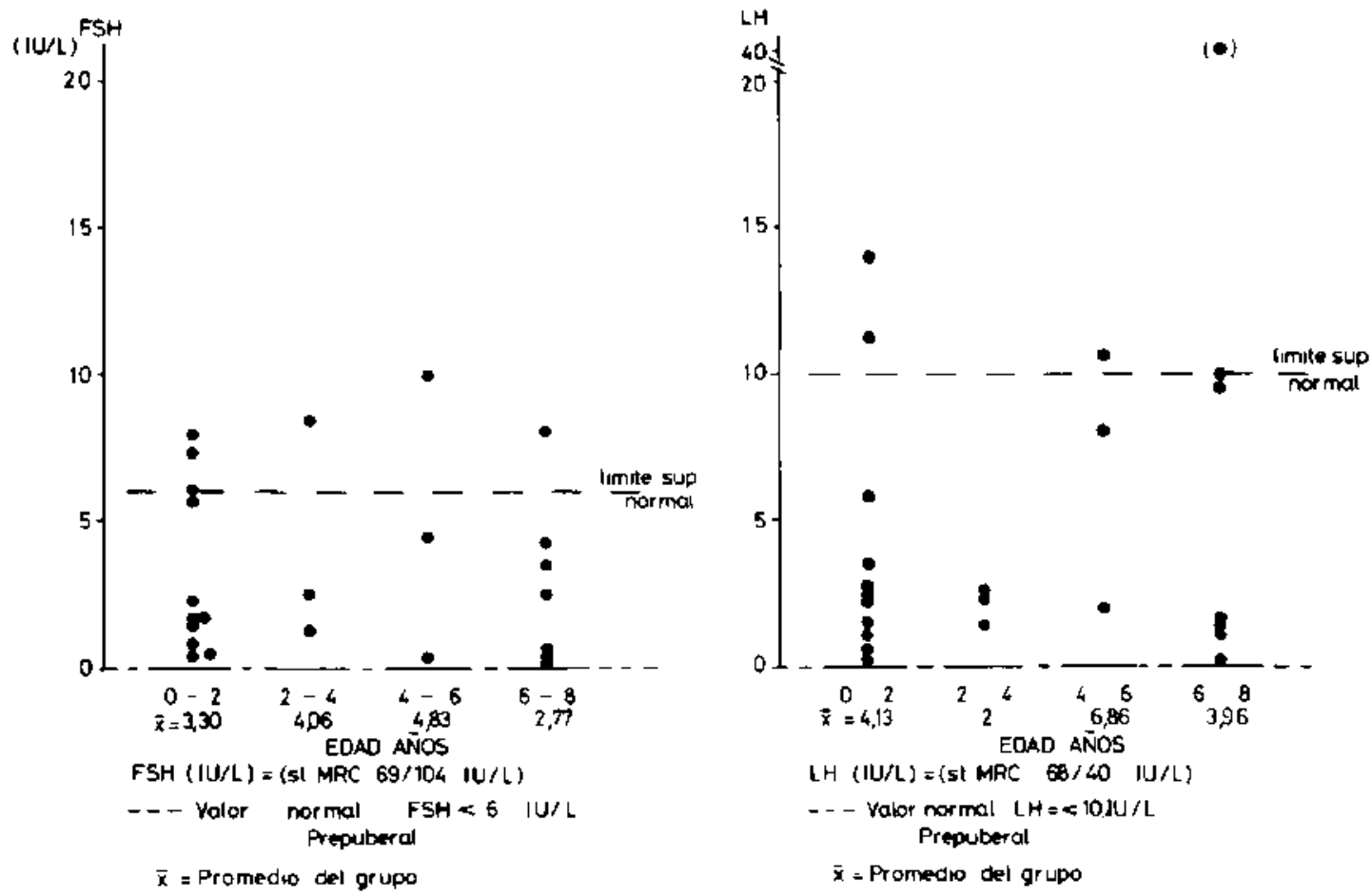

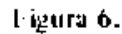

Valores plasmaticos de ISSII y $\mathrm{LH}$ en 24 mithas con Telarauia l'rematura. Línea horizontal (-- ) límite superior prepuberal nomal. $\overline{\mathrm{X}}=$ Valor pronedio del grupo etario.

En 17 casos se midieron Gonadotrofinas (G.T.) biológicas en la orina de 24 hrs. sin encontrar correlación entre ellas y los valores de FSH o LH (coeficiente de correlación lineal para FSH y G.T. $=0,26$; para $\mathrm{LH}$ y $\mathrm{GT}=-0, \mathrm{I}$ (69).

En 8 pacjentes se efectuaron mediciones plasmáticas de prolactina obteniendo valores dentro del rango normal ${ }^{15}$.

En todos los casos el EEG y fondo de ojo fueron normales.

En todas las pacientes se estudió la velocidad de crecimiento en relación con el incremento de la edad ósea. En la mayoría fué rormal. En dos casos se encontraron velocidades de crecimiento más lentas. En el grupo de riñas mayores se apreció un desplazamiento del período de crecimiento rápido hacia edades más tempranas que no tuvo significación estadistica.

\section{DISCUSION}

Nuestros casos de telarquia prematura se distribuyeron preferentemente entre los menores de 2 años y las niñas de 6 a 8 años.

En las publicaciones revisadas no hemos podido constatar similar hallazgo, tal vez porque todas ellas cuentan con una muestra reducida de

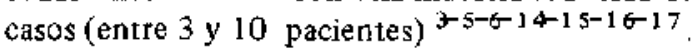

Entre las hipótesis que podrían plantearse para explicar este lallazgo están el mayor control programático de ambos grupos etarios, los primeros pur el programa materno infantil y los programas escolares en los segundos y cabe también la posibilidad que durante estos períodos exista diferente sensibilidad del eje hipotálamo-hipófisis-ovario que pudiese determinar, en menores de 2 años $y$ mayores de 6 , la apari. ción precoz de telarquia. Algunos autores des. criben valores elevados de FSH en el primer año de vida $y$ en la etapa pre-puberal, precediendo un año a la elevación de L.H. s-6. $^{\text {. }}$

El estrogenismo vaginal es un hallazgo frecuente en la telarquia prematura ${ }^{\mathfrak{3 - 2}-10-6}$. En nuestra casuística se presentó en $58 \%$ de los casos. Es conocido que la telarquia prematura puede atribuirse a estrógenos secretados por folículos de Graaf, especialmente aquellos que llegan a ser quisticos y luteinizados. Se han descrito con cierta frecuencia, durante la infancia, diferentes grados de maduración folicular en foliculos primordiales; en cambio la telarquia prematura es un hallazgo relativamente poco común, de tal manera que pare. cería que sólo los folículos que alcanzan cierta etapa crítica de maduración o tamaño tendrían capacidad de secretar una cantidad de 
estrógenos suficiente para provocar algún grado de desarrollo mamario. Un efecto transitorio ejercido por el folículo ovárico luteinizado, podría explicar la presencia aislada de estrogenismo en el epitelio vaginal. Sin embargo, hay autores que han logrado detectar concentraciones significativas de estradiol plasmático en pacientes con telarquia prematura ${ }^{2-4}$. Nosotros no encontramos relación entre el grado de estrogenismo vaginal, la evolución de la telarquia y la magnitud del desarrollo mamario. Esto concuerda con lo descrito por otros autores ${ }^{6-2-4}$ y podría deberse a diferentes sensibilidad de los efectores mamario y vaginal.

En la mayoría de nuestros pacientes la E.O. se encontró en el rango normal, hecho también descrito en otros trabajos $s^{6-15-18}$. De las pacientes con E.O. adelantada, algunas presentaron evolución regresiva o estacionaria de la telarquja, y otras progresiva. La aceleración de la E.O. podría corresponder а uл patrón genético điferen. te o ser un signo de pubertad iniciada más temprano: sin embargo, ninguno de nuestros casos con telarquia prematura evoluciono hacia pubertad precoz.

El estudio de los valores de FSH y LH plasmático mostró aumentos en pocos casos y casi siempre de pequeña magnitud. Diferentes auto$\mathrm{res}^{2-5-6-17}$ han obtenido resultados dispares: Algunos $^{6}$ reportaron valores basales elevados de FSH e hipenespuesta de ésta a LHRH. Otros encontraron cifras basales normales ${ }^{2-5-17}$, con respuesta en concentraciones prepuberales de LH a LHRH y tendencia a mayor respuesta de FSH a LHRH ${ }^{5}$. Estos últimos han sugerido que podría ser de utilidad realizar la prueba de $\mathrm{LHRH}$ para el diagnóstico diferencial entre pubertad. precuz (P.P.) en etapas iniciales y telarquia pre. matura. Ел ta P.P. se encuentra un aumento significativo de la respuesta de LH a la estimulación con LHRH, lo que no ocurre en la telarquia prematura. En nuestros pacientes no pudimos realizar esta prueba $y$ creemos que en el futuro sería interesante aplicarla.

Del estudio de nuestros pacientes podemos concluir que la telarquia prematura aparen. temente no condiciona una alteración de la velocidad de crecimiento, ya que si bien en las niñas mayores se observó un desplazamiento del periodo de crecimiento rápido hacia edades más tempranas, éste no tiene significado estadístico.

\section{RESUMEN}

Se estudiaron 48 casos de telarquia prematura cuyas edades de consulta fluctuaron entre 1 mes y 8 años. Se realizó historia clínica, examen an- tropométrico, evaluación de grado de desarrollo mamario (según Tanner) y exámenes de Laboratorio: Rx. de cráneo E.O., EEG., fondo de ojo, $17 \mathrm{ks}, 170 \mathrm{H}$, y GT en orina 24 hrs., Colpocitograma, FSH, LH y PRL plasmática por RIA. Las pacientes fueron observadas por períodos de 3 meses a 6 años 6 meses, con un promedio de 2 años 6 meses. Se encontró relación entre desarrollo mamario y la presencia de estrogenismo vaginal en más del $50 \%$ de los casos. La acelera. ción de la edad ósea fué un hallazgo poco frecuente. Las concentraciones de FSH y LH plasmáticas estaban en la mayoría de los casos dentro del rango normal. No se observaron modificacio. nes significativas de la velocidad de crecimiento ni progresión de la telarquia prematura a pubertad precoz.

\section{REFERENCLAS}

1 wilkins L., The Diagnosis and treatment of Endoctithe Disotders in chilhood and Adolescence 3 rd Ed. Springfield, Charles C. Thomas, 1965 pp 206 7 .

2 Jenner M.R., Kelch R.P., Kapian S.L., Grumbach, M.M.: Hormonal Chages in puberty: IV Plasma estradiol, LH.FSH iл prepubertal children, puberal remales, and in precociou puberty, premature thelarche, bypogonadism, and in a child with a feminizing ovarian tumor. J. Clin. Endocrinol Metab. 34: 521, 1972.

3 Collet-Solberg P.R., Grumbach M.M.A.: A simplified procedure for evaluating estrogenic effects and the sex chromatin pathern in exfoliated cells in urine: Studies in premature thelarche and Gynecomastia of adolescence. J. Pediat $1.66: 883,1965$.

4 Escobar ME. Rivarola Bengadá C.: Plasma concen. tration of estradiol $17 \beta$ in premature thelarche and in different types of sexual precocity. Acta Endacrinol 81: 351,1976 .

5 Reiter E.O., Kaplan S.L., Conte F.A., and Grumbach M.M.: Responsivity of Pituitary Gonadotropes of Luteinizing Hormone-relea sing Factor in Idiopathic Precocious Puberty, Precocious Thelarche, Precocious Adrenarche, and in Patients Treated with Meroxiprogesterone Acetato. Pediatrics 9: 111, 1975.

${ }^{6}$ Pasquino A.M., Piccolo F., Scalamandre A., et al Hypothalamic-pituitary-gonadotropic function in girls with premature thelarche. Arch dis Child 55: $941,1980$.

7 Marshall W.A., and Tonner J.W.: Variations in Pattem of pubertal changes in Girls. Arch. Dis. Child. 44: 291,1969 .

${ }^{8}$ Greulich $V . W$ and Pyle, S.Y.: Atlas of Skeletal Development of the Hand and $W_{\text {rist }} 2$ nd Ed Stanford Lniversity Press. 1955.

9 Koufman R.H.Leed, $L, J$ : Cervical and vaginal cy toloBy in the child and adolescent. Pediatr. Clin. North Am. 19: 547, 1972.

10 Preeyasombet Ch., and Kenny, F.: Urocitograms in normal children and various abnormal conditions. Pediatrics 38: 436, 1966.

11 Novak Edmund $R$. Tratado de Ginecología 90 Ed, México, Interamericana, 1977 pp 729-753, 
12 Avendaño A., Neira M., Smith S., Et, al. Edades óseas de escolares fiscales del Area Hospitalaria Nor. te de Santjago, según percentiles de estatura. Pediatría $17: 23,1974$.

13 Bossi E.E., Joss and Zurbrugg R.P.: Evaluation of the effectivenesssof Treatment on Adult Heigth Prognosis in disorders with advances and Retarded Bone Age. Acta Pediatric. Scand. 62: 401, 1973.

14 Kenny F.M., Midgley A.R., Jaffe R.B., et al.: Radioinmuno assayable serum $L H$ and FSH in girls with sexual Precotiy, premature thelarche and adrenarche. J. Clin. Endocrinol. Metab, 29; 1272, 1969.

15 Gaufriez A., Wolter $R .$, Govaerts M., eta al Gonadotropins and prolactin pituitary reserve in premature therlarche J. Pediatric 91: 751, 1977.

16 Sánchez $F$, Forsbach $C$., Zárate $A$., et. al. Cunical use of the LH-RH in assessing gonadotrpoic reserve in children with idiophatic precocious puberty, premature thelarche and premature adrenarche. Arch Invest Med. (Mex) 7: 123, 1976.
${ }^{17}$ De Sanctis C. LALA R, Doghiani P. et. al. Dirámica funzionale delle gonadotropine in bambine con pubertá precoce idiopatica, con pubarca e Telarca prematuro, Minerva Pediatr. 29: 2425, 1977.

18 Luchy A.W., Rich $B H$, Rosenfiel RL Bioactive LH: A test to discriminate true precocious puberty from premature thelarche and Adrenarche. J. Pediatr. 97: 214,1980 .

19 Spieget MR., Theory and problems of statitics N. York. Schaum publishing company, 1961 pp. 241244.

20 Vivanco $X$., Abodorsky $N$., Determinaciones de FS $y$ LH en niños sanos de diferentes edades. Cornunica. ción personal.

21 Silber, R.H., Porter, C.C.: Determinations of 17, 21 dihydroxy-20 - Ketosteroide in urine and plasma. J. Biol. chem. $210: 923,1954$.

22 Brener, $H$. Methods of hormone analysis. Stuttgart Germany 1976, pp 189-194.

23 M.R.C. Committee on Clinical endocrinology. Proposed standar method of 17 - Ketosteroid determination. Lancet 2: $\$ 85,1951$. 